\title{
A SPECTRAL SEQUENCE FOR ORBIFOLD COBORDISM
}

\author{
ANDRÉS ÁNGEL \\ Department of Mathematics, Stanford University \\ Stanford, CA 94305, U.S.A. \\ E-mail: jangel@math.stanford.edu
}

\begin{abstract}
The aim of this paper is to introduce a spectral sequence that converges to the cobordism groups of orbifolds with given isotropy representations. In good cases the $E^{1}$-term of this spectral sequence is given by a certain cobordism group of orbibundles over purely ineffective orbifolds which can be identified with the bordism group of the classifying space of the Weyl group of a finite subgroup of $O(n)$. We use this spectral sequence to calculate some cobordism groups of orbifolds for low dimensions, and in particular, we show that every three dimensional effective oriented orbifold, or even only locally oriented orbifold, bounds. And although every two dimensional effective oriented orbifold bounds, $\mathbb{R P}^{2}$ is the generator of the second cobordism group of locally oriented orbifolds.
\end{abstract}

Introduction. Orbifolds, originally introduced as $V$-manifolds by Satake, and named this way by Thurston, are useful generalizations of manifolds, locally they look like the quotient of euclidean space by the action of a finite group. Orbifolds have been used for some time in geometry and topology and provide a broad example of objects with inner symmetry, and therefore, their appearance and usefulness in moduli problems like Gromov-Witten theory. Recently they have gained more popularity due to their use in theoretical physics. Their "stringy" properties have just started to be understood and the purpose of this work is to contribute to the study of orbifolds from the classical realm of algebraic topology by adapting techniques from equivariant cobordism.

In [5], K. Druschel started the study of the cobordism groups of effective oriented orbifolds by introducing a complete set of invariants that determine the oriented cobordism class up to torsion. These generalized Pontrjagin numbers are used, for example, to prove that any odd dimensional oriented effective orbifold rationally bounds, and unlike in the manifold case, there is a $4 k+2$ dimensional oriented effective orbifold that does not bound rationally. As in the nonequivariant case, these numbers are used to give generators for

2000 Mathematics Subject Classification: Primary 57R90, 57N70.

Key words and phrases: orbifolds, cobordism.

The paper is in final form and no version of it will be published elsewhere. 
the rational orbifold cobordism ring as a free algebra over $\Omega_{*} \otimes \mathbb{Q}$. To study the torsion, K. Druschel in [6] considers cobordism groups with restrictions on the set of local groups to show that every two and three dimensional effective oriented orbifold bounds.

Generalizing techniques introduced by Conner and Floyd [3], we construct a spectral sequence that can be used to calculate cobordism groups of orbifolds. In low dimensions, the knowledge of the finite groups that can appear as isotropy groups of orbifolds, allow us to give geometric arguments to calculate some of these cobordism groups, in particular we can explain K. Druschel's results on three dimensional oriented orbifolds as the collapse of a spectral sequence at the $E^{2}$-level.

A complete description of the cobordism ring of oriented or locally oriented orbifolds is still unknown, but when we consider orbifolds with only isotropy groups of odd order, there is a complete description of the corresponding ring in terms of usual bordism theory [1].

This paper is organized as follows. In section one we describe the necessary background on orbifolds. Section two introduces the main calculational tools, families of local representations and cobordism groups of orbifolds with restricted local representations. In the spirit of [3], these cobordism groups with restricted local representations fit into a long exact sequence. By adding extra conditions on our families, the relative term in this long exact sequence can be computed in terms of usual bordism theory. By constructing an exhausting sequence of families, our spectral sequence is introduced.

By analyzing the differentials of this spectral sequence we obtain that in dimension two and three it collapses at the $E^{2}$-level. From this, and the fact that every two and three dimensional oriented manifold bounds, we obtain the corresponding result for effective oriented orbifolds. Following similar arguments we conclude that every three dimensional effective locally oriented orbifold bounds, and that $\mathbb{R} \mathbb{P}^{2}$ represents the only non-trivial cobordism class of locally oriented two dimensional effective orbifolds.

Acknowledgements. The author would like to thank Bernardo Uribe and Ernesto Lupercio for their interest and helpful conversations about this work, the organizers of the Algebraic Topology: Old and New; M. M. Postnikov Memorial Conference, Będlewo, Poland June 2007 for the opportunity to present some of the results mentioned in this work. The results presented here are part of the author's dissertation under the direction of Professor Ralph Cohen to whom the author owes many hours of guidance and suggestions.

This paper is dedicated to the memory and family of Hildebrando Urdinola whose tragic death took us by surprise and who we all remember with love.

1. Preliminaries. The building blocks of orbifolds are representations.

Notation 1. Given an $n$-dimensional orthogonal representation $(G, \rho)$ of a finite group $G$, where $\rho$ denotes the corresponding homomorphism $G \rightarrow O(n)$, we denote by $V(\rho), \mathbb{R}^{n}$ with the action given by $\rho$, and by $S(\rho)$ and $D(\rho)$ the corresponding sphere and disk. The representation determines a conjugation class in $O(n)$ and when the representation is faithful, i.e. $\rho$ is injective, if no confusion arises, we will identify $G$ with a subgroup of $O(n)$. 
Let $X$ be a paracompact Hausdorff topological space.

Definition 1.1. An $n$-dimensional orbifold chart on $X$ is a triple $(\bar{U}, G, U)$, where $\bar{U}$ is a manifold, $G$ is a finite group acting smoothly on $\bar{U}$ and $U$ is an open subset of $X$, homeomorphic to $\bar{U} / G$. We call $\pi$ the natural projection $\pi: \bar{U} \rightarrow \bar{U} / G \cong U$.

Definition 1.2. An embedding of charts

$$
(\bar{U}, G, U) \hookrightarrow(\bar{V}, H, V)
$$

is a differentiable embedding $\bar{U} \hookrightarrow \bar{V}$ that is equivariant with respect to a monomorphism $G \hookrightarrow H$, that preserves the kernel of the actions.

Definition 1.3. Two charts $(\bar{U}, G, U) \hookrightarrow(\bar{V}, H, V)$ are compatible if for every point in $U \cap V$, there exists a chart $(\bar{W}, K, W)$ with embeddings of charts

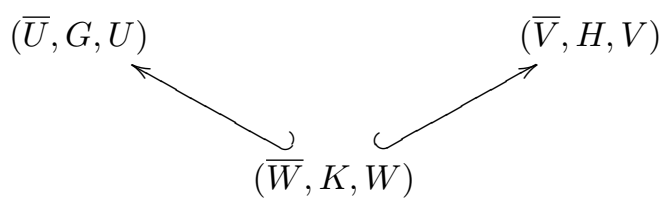

Definition 1.4. An orbifold atlas on $X$ is a family of charts $\left(\bar{U}_{\alpha}, G_{\alpha}, U_{\alpha}\right)$ that is compatible and covers $X$. An orbifold structure on $X$ is just an equivalence class of orbifold atlases, where two atlases are equivalent if there is a zig-zag of common refinements ${ }^{1}$. We will denote an orbifold structure on $X$ by calligraphic letters, like $\mathcal{X}$, and the topological space $X$ will be called the underlying space and will be denoted by $|\mathcal{X}|$.

EXAMPLE 1.5. A manifold $M$ with an action of a finite group $G$ gives rise to an orbifold that we will denote by $[M / G]$, it has an atlas with only one chart. In a more general vein, if a compact Lie group acts on a manifold with finite stabilizers, then the quotient space can be endowed with an orbifold structure. Charts can be constructed with the slice theorem for differentiable actions.

Around each point we can linearize the actions by using the exponential map, and therefore any orbifold has an atlas consisting of linear charts, by which we mean, charts of the form $\left(\mathbb{R}^{n}, G, U\right)$, where $G$ acts on $\mathbb{R}^{n}$ via an orthogonal representation $\rho: G \hookrightarrow O(n)$ and $\mathbb{R}^{n} / G \cong U$.

Given a point $x \in X$, take a chart $(\bar{U}, G, U)$ around $x$, let $\bar{x} \in \bar{U}$ be a lift of $x$. Then we define the isotropy group of $x$ to be,

$$
G_{x}=\{g \in G \mid g \bar{x}=\bar{x}\}
$$

Different lifts of $x$ give conjugate subgroups of $G$ and different choices of charts give isomorphic groups. A point $x$ such that $G_{x} \neq\{e\}$ is called a singular point, and an orbifold without singular points is just a manifold.

Again, by the slice theorem for smooth actions, we have at each point a well defined representation of the isotropy group $G_{x}$ that gives a chart around $x$. We call this representation $\left(G_{x}, \rho_{x}\right)$, the local representation at $x$.

\footnotetext{
${ }^{1}$ I would like to thank Yael Karshon for pointing out to me the subtleties of this part of the definition [8].
} 
A local orientation of an orbifold is a choice of an orientation of $V\left(\rho_{x}\right)$ that makes the action of $G_{x}$ orientation preserving, this induces orientations on all smaller charts. This is equivalent to identifying $G_{x}$ with a subgroup of $S O(n)$. As with manifolds, an orientation is just a choice of local orientations in such a way that the transition functions are orientation preserving. For an oriented orbifold, the local representation gives a map $\rho: G_{x} \hookrightarrow S O(n)$, well defined up to conjugation by elements of $S O(n)$.

2. Families of local representations. In the early sixties Conner and Floyd showed the effectiveness of bordism methods in the analysis of transformation groups. In their monograph [2] and in a later paper [3], they introduced a framework for the study of actions of finite groups on compact manifolds. The basic idea is to analyze the cobordism groups by the information provided by fixed points and normal bundles. Their main calculational tools are families of subgroups and fixed point homomorphisms.

In this section we adapt these techniques and introduce families of local representations, cobordism groups of orbifolds with restricted local representations and a long exact sequence relating them. This is much in the spirit of [3] and [9].

Recall that a family of subgroups of $G$ is a collection of subgroups that is closed under conjugation and subgroups. The analogous notion that we think is the correct generalization to orbifolds is,

Definition 2.1. A family of local representations $\mathcal{F}$ is a collection of finite dimensional representations of finite groups that satisfies the following conditions:

i) It is closed under isomorphism of representations.

ii) If $(G, \rho) \in \mathcal{F}$ is a representation of the finite group $G$, then all the representations $\left(G_{x}, \rho_{x}\right)$ of the isotropy groups for $x \in[V(\rho) / G]$ belong to $\mathcal{F}$.

iii) $(G, \rho) \in \mathcal{F}$ if and only if $(G, \rho \oplus i d) \in \mathcal{F}$, where $\rho \oplus i d$ is the direct sum of $\rho$ with the trivial representation.

A family of local representations comes with a natural partial order $(H, \tau) \leq(G, \rho)$ if and only if $(H, \tau)$ appears among the local representations of the linear orbifold $[V(\rho) / G]$. In particular $H$ is isomorphic to a subgroup of $G$, and $\rho \mid H=\tau$.

REMARK 2.2. The intersection of families is a family, and every collection of representations is contained in a family. Therefore we can talk about the family generated by a set of representations.

We do not require the families to be closed under restrictions for subgroups, just under the partial order described above, this is enough for our purposes and makes the calculations easier.

By property iii) a family $\mathcal{F}$ has elements in each dimension, we denote by $\mathcal{F}(n)$ the $n$-dimensional elements of $\mathcal{F}$.

Cobordism groups of orbifolds with restricted local representations. Now we will introduce cobordism groups with given local representations. The main theme of this work is to study the change in the cobordism groups when we increase the allowed local representations. 
Definition 2.3. Given two families of local representations $\mathcal{F} \supseteq \mathcal{F}^{\prime}$, we say that $\mathcal{X}$ is an $\left(\mathcal{F}, \mathcal{F}^{\prime}\right)$-orbifold if every local representation of $\mathcal{X}$ belongs to $\mathcal{F}$, and all local representations of points on the boundary belong to $\mathcal{F}^{\prime}$.

Condition iii) of definition 2.1 tells us that if $\mathcal{X}$ is an $\mathcal{F}$-orbifold, then $\mathcal{X} \times I$ is also, and that the boundary of an $\left(\mathcal{F}, \mathcal{F}^{\prime}\right)$-orbifold is an $\mathcal{F}^{\prime}$-orbifold. For example if $[V(\rho) / G]$ is a $\mathcal{F}$-orbifold then $[D(\rho) / G]$ and $[S(\rho) / G]$ are also $\mathcal{F}$-orbifolds.

Definition 2.4. We say that an $\left(\mathcal{F}, \mathcal{F}^{\prime}\right)$-orbifold $\mathcal{X}^{n}$ bounds, if there exists $\mathcal{W}^{n+1}$, an $(\mathcal{F}, \mathcal{F})$-orbifold with $\mathcal{X}_{1}^{n}$, an embedded, $\left(\mathcal{F}, \mathcal{F}^{\prime}\right)$-orbifold, such that $\mathcal{X}_{1}^{n}$ and $\mathcal{X}^{n}$ are orbifold diffeomorphic, and such that for all $x \in \partial W^{n+1} \backslash \mathcal{X}_{1}^{n},\left(G_{x}, \rho_{x}\right) \in \mathcal{F}^{\prime}$. If the orbifolds are oriented we require $\mathcal{X}_{1}^{n}$ to have the induced orientation from $\mathcal{W}^{n+1}$.

We say $\mathcal{X}^{n}$ is cobordant to $\mathcal{X}_{1}^{n}$ if the disjoint union $\mathcal{X}^{n} \cup-\mathcal{X}_{1}^{n}$ bounds.

The necessary tools from differential topology, like the existence of collaring neighborhoods, the glueing by orientation reversing diffeomorphisms of open sets and the straightening angles, are available, and as in the equivariant case, we have that cobordism defines an equivalence relation on the class of $\left(\mathcal{F}, \mathcal{F}^{\prime}\right)$-orbifolds.

Definition 2.5. We define $\mathcal{O}_{n}\left(\mathcal{F}, \mathcal{F}^{\prime}\right)$ to be the group of cobordism classes of $n$ dimensional oriented and effective $\left(\mathcal{F}, \mathcal{F}^{\prime}\right)$-orbifolds under the operation of disjoint union.

If $\mathcal{F}^{\prime}$ is empty, $\mathcal{O}_{n}\left(\mathcal{F}, \mathcal{F}^{\prime}\right)$ is the group of cobordism classes of closed orbifolds where all local representations are in $\mathcal{F}$, we denote this group by $\mathcal{O}_{n}(\mathcal{F})$, the $\mathcal{F}$-orbifolds. In particular if $\mathcal{F}$ is the trivial group, we have the cobordism classes of all manifolds.

Every $\mathcal{F}^{\prime}$-orbifold is an $\mathcal{F}$-orbifold, and similarly every $\mathcal{F}$-orbifold is an $\left(\mathcal{F}, \mathcal{F}^{\prime}\right)$ orbifold. Together with taking boundary we have the natural maps,

$$
\begin{aligned}
\mathbb{I}: \mathcal{O}_{n}\left(\mathcal{F}^{\prime}\right) & \rightarrow \mathcal{O}_{n}(\mathcal{F}), \\
\mathbb{L}: \mathcal{O}_{n}(\mathcal{F}) & \rightarrow \mathcal{O}_{n}\left(\mathcal{F}, \mathcal{F}^{\prime}\right), \\
\partial: \mathcal{O}_{n}\left(\mathcal{F}, \mathcal{F}^{\prime}\right) & \rightarrow \mathcal{O}_{n-1}\left(\mathcal{F}^{\prime}\right),
\end{aligned}
$$

which fit in a long exact sequence,

$$
\cdots \rightarrow \mathcal{O}_{n}\left(\mathcal{F}^{\prime}\right) \rightarrow \mathcal{O}_{n}(\mathcal{F}) \rightarrow \mathcal{O}_{n}\left(\mathcal{F}, \mathcal{F}^{\prime}\right) \rightarrow \mathcal{O}_{n-1}\left(\mathcal{F}^{\prime}\right) \rightarrow \cdots
$$

The point of this construction is that in good cases it is possible to identify the relative term of this long exact sequence with some cobordism group of orbibundles.

An element $\mathcal{X} \in \mathcal{O}_{n}\left(\mathcal{F}, \mathcal{F}^{\prime}\right)$ is zero if and only if it is an $\mathcal{F}$-orbifold and there is an $\mathcal{F}^{\prime}$-orbifold, with the same boundary, in such a way that when you glue them along the common boundary they form the boundary of an $\mathcal{F}$-orbifold. In particular if $\mathcal{X}$ does not have boundary, then it is zero if and only if it is cobordant to an $\mathcal{F}^{\prime}$-orbifold with a cobordism that uses only elements in $\mathcal{F}$. For example,

LEMmA 2.6. Let $\mathcal{X}^{n}$ be an $\left(\mathcal{F}, \mathcal{F}^{\prime}\right)$-orbifold, let $\mathcal{X}_{1}^{n}$ be a compact orbifold, regularly embedded in the interior of $\mathcal{X}^{n}$. Suppose that all the isotropy groups of points $x \in \mathcal{X}^{n} \backslash \mathcal{X}_{1}^{n}$ belong to $\mathcal{F}^{\prime}$. Then $\mathcal{X}^{n}=\mathcal{X}_{1}^{n}$ in $\mathcal{O}_{n}\left(\mathcal{F}, \mathcal{F}^{\prime}\right)$. 
Reduction to the singular set. Now suppose that $(H, \rho)$ is an element of $\mathcal{F}$, we denote by $\mathcal{X}^{\rho}$, the $\rho$-singular set of $\mathcal{X}$,

$$
\mathcal{X}^{\rho}=\left\{x \in \mathcal{X} \mid(H, \rho) \leq\left(G_{x}, \rho_{x}\right)\right\}
$$

This is the analogue of fixed points, but in general $\mathcal{X}^{\rho}$ is not necessarily an orbifold, due to the fact that there could be different subgroups of $G_{x}$ isomorphic to $H$ that are conjugate in $O(n)$. Therefore $\mathcal{X}^{\rho}$ is only a union of suborbifolds. But when $\rho$ is maximal in the local representations of $\mathcal{X}$,

$$
\mathcal{X}^{\rho}=\left\{x \in \mathcal{X} \mid(H, \rho) \simeq\left(G_{x}, \rho_{x}\right)\right\}
$$

is an orbifold. Charts can be given in the following way: for $x \in \mathcal{X}^{\rho}$ and linear chart $\left(\bar{U}_{x}, G_{x}\right)$ of $\mathcal{X}$ compatible with the orientation, take charts, $\left(\left(\bar{U}_{x}\right)^{G_{x}}, G_{x}\right)$ with $G_{x}$ acting trivially. Where $\left(\bar{U}_{x}\right)^{G_{x}}$ is the fixed point subspace of $G_{x}$. The change of coordinate maps for $\mathcal{X}^{\rho}$ is given by restricting the overlap maps for $\mathcal{X}$.

If $\mathcal{X}$ is compact, the maximality of $\rho$ implies that $\mathcal{X}^{\rho}$ is also compact. Now we will define the normal orbivector bundle $\nu_{\rho} \rightarrow \mathcal{X}^{\rho}$. Given $x \in \mathcal{X}^{\rho}$, and a linear chart $\left(\bar{U}_{x}, G_{x}\right)$ of $\mathcal{X}$ around $x$, then

$$
\left.T \bar{U}_{x}\right|_{\left(\bar{U}_{x}\right)^{G x}}=T\left(\bar{U}_{x}\right)^{G_{x}} \oplus V_{x}
$$

where $V_{x}$ is the normal bundle of $\left(\bar{U}_{x}\right)^{G_{x}}$ in $\bar{U}_{x}$, since we are using linear charts we can identify $V_{x}$ with the trivial bundle $\left(\bar{U}_{x}\right)^{G_{x}} \times\left(\bar{U}_{x}\right)^{G_{x} \perp}$. Note that this bundle has an action of $G_{x}$ that covers the trivial action on $\left(\bar{U}_{x}\right)^{G_{x}}$. We can patch these bundles using the overlap maps from $\mathcal{X}$ to get the normal orbivector bundle $\nu_{\rho} \rightarrow \mathcal{X}^{\rho}$.

As representations, $\rho$ decomposes into a sum $\hat{\rho} \oplus \underbrace{i d \oplus \cdots \oplus i d}_{k}$, where $\hat{\rho}$ has no trivial summands and $k$ is the multiplicity of the trivial representation, i.e. the dimension of the subspace fixed under the action of $G, k$ is called the degree of the representation and therefore the rank of the orbivector bundle $\nu_{\rho}$ is $n-k$.

The decomposition $\rho \cong \hat{\rho} \oplus \underbrace{i d \oplus \cdots \oplus i d}_{k}$ induces a factorization

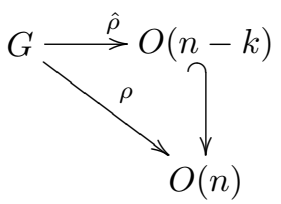

where $O(n-k) \rightarrow O(n)$ is the natural inclusion.

Now, a calculation shows that the structure group of the normal orbibundle $\nu_{\rho}$ reduces to $N_{O(n-k)} \hat{\rho}(H)$, the normalizer in $O(n-k)$ of the image of $\hat{\rho}: G \rightarrow O(n-k)$. Summarizing

THEOREM 2.7. Given an $n$-dimensional compact orbifold $\mathcal{X}$, and a maximal element, $(H, \rho)$ of the local representations, the $\rho$-singular set, $\mathcal{X}^{\rho}$ is a compact suborbifold of $\mathcal{X}$ and the normal bundle $\nu_{\rho} \rightarrow \mathcal{X}^{\rho}$ is an orbivector bundle with structural group $N_{O(n-k)} H$, where $k$ is the dimension of the fixed points subspace of $\mathbb{R}^{n}$ under the action of $H$. Even more, $\mathcal{X}^{\rho}$, is a purely ineffective orbifold [7] with trivial stabilizer $H$. 
The following theorem follows from the previous theorem, the existence of tubular neighborhoods and lemma 2.6. It is the key result of this section.

THEOREM 2.8. Suppose that $\mathcal{F} \supset \mathcal{F}^{\prime}$ are families which differ at dimension $n$ by the representation $(H, \rho)$, i.e. $\mathcal{F}(n)-(H, \rho)=\mathcal{F}^{\prime}(n)$. Then the oriented cobordism group $\mathcal{O}_{n}\left(\mathcal{F}, \mathcal{F}^{\prime}\right)$ is isomorphic to the cobordism group of orbibundles over purely ineffective orbifolds, where the orbibundles have an oriented total space, the fiber representation is the non-trivial summand of $\rho$ and the $\rho$-singular set is the zero section.

For a local represenation $(H, \rho)$ not necessarily maximal, we can still define

$$
\mathcal{X}^{\rho}=\left\{x \in \mathcal{X} \mid(H, \rho) \simeq\left(G_{x}, \rho_{x}\right)\right\}
$$

and we have a similar statement, but due to the lack of maximality we do not have compactness.

Since $\mathcal{X}^{\rho}$ is purely ineffective orbifold, the underlying space is a manifold. The transition functions of the orbivector bundle $\nu_{\rho}$ are elements of $N_{O(n-k)} \hat{\rho}(H)$ defined up to elements of $H$, now $N_{O(n-k)} \hat{\rho}(H)$ acts on $\mathbb{R}^{n-k}$ and $N_{O(n-k)} \hat{\rho}(H) / \hat{\rho}(H)$ acts effectively on $\mathbb{R}^{n-k} / H$, therefore, we can use the same set of transition functions, seen as elements of $N_{O(n-k)} \hat{\rho}(H) / \hat{\rho}(H)$, to construct an $N_{O(n-k)} \hat{\rho}(H) / \hat{\rho}(H)$-principal bundle over the underlying space, which is a manifold.

REMARK 2.9. For $\mathcal{X}$, an effective orbifold, the orbibundle $\nu^{\rho} \rightarrow \mathcal{X}^{\rho}$ is determined by the associated principal $N_{O(n-k)} \hat{\rho}(H) / \hat{\rho}(H)$-bundle over the underlying space $\left|\mathcal{X}^{\rho}\right|$ and the principal $H$-bundle $N_{O(n-k)} \hat{\rho}(H) \rightarrow N_{O(n-k)} \hat{\rho}(H) / \hat{\rho}(H)$.

If the original orbifold is oriented, then we do not necessarily have that the singular set is oriented, or that the normal bundle is oriented, we only have that $T \mathcal{X}^{\rho} \oplus \nu_{\rho}$ is oriented. The transition functions for the normal bundle and the overlap maps for the singular set come from the restriction of these transition functions to subspaces and that is were the orientability can be lost. However, in the case when the representations have degree zero, the total space of the normal bundle is oriented, and the structure group is trivial.

Now we come to the main results of this section. When the families are close enough, the relative term in the long exact sequence becomes computable in terms of the bordism group of the classifying space of the Weyl groups of the representations in $\mathcal{F}-\mathcal{F}^{\prime}$.

THEOREM 2.10. Suppose that $\mathcal{F} \supset \mathcal{F}^{\prime}$ are families which differ at dimension $n$ by the representation $(H, \rho)$, i.e. $\mathcal{F}(n)-(H, \rho)=\mathcal{F}^{\prime}(n)$. Then the oriented cobordism group $\mathcal{O}_{n}\left(\mathcal{F}, \mathcal{F}^{\prime}\right)$ is isomorphic to the twisted cobordism group $\Omega_{k, t}\left(B N_{O(n-k)} \hat{\rho}(H) / \hat{\rho}(H)\right)$ of pairs $(M, f)$, where $M$ is a closed compact $k$-dimensional manifold and $f: M \rightarrow$ $B N_{O(n-k)} \hat{\rho}(H) / \hat{\rho}(H)$ is a map such that $T M \oplus f^{*} \Gamma$ is oriented. $\Gamma$ is the universal $N_{O(n-k)} \hat{\rho}(H) / \hat{\rho}(H)$ bundle over $B N_{O(n-k)} \hat{\rho}(H) / \hat{\rho}(H)$.

Proof. By theorem 2.8, the relative group $\mathcal{O}_{n}\left(\mathcal{F}, \mathcal{F}^{\prime}\right)$ can be identified with a cobordism group of orbibundles over purely ineffective orbifolds, where the orbibundles have an oriented total space, the fiber representation is the non-trivial summand of $\rho$ and the $\rho$-singular set is the zero section. Moreover these orbibundles have structural group $N_{O(n-k)} \hat{\rho}(H)$, and we can consider an associated principal $N_{O(n-k)} \hat{\rho}(H) / \hat{\rho}(H)$-bundle 
over the underlying space. Since the underlying space of a purely ineffective orbifold is a manifold, by classifying this principal bundle we get an element of

$$
\Omega_{k, t}\left(B N_{O(n-k)} \hat{\rho}(H) / \hat{\rho}(H)\right)
$$

where the twisting comes from the fact that we are only requiring the total space of the orbibundle to be oriented.

If $N_{O(n-k)} \hat{\rho}(H) \leq S O(n-k)$ then, $\nu_{\rho}$ is an oriented orbivector bundle and from the orientation of $\mathcal{X}$ we get an orientation of $\mathcal{X}^{\rho}$ thus

$$
\mathcal{O}_{n}\left(\mathcal{F}, \mathcal{F}^{\prime}\right) \simeq \Omega_{k}\left(B N_{O(n-k)} \hat{\rho}(H) / \hat{\rho}(H)\right)
$$

and we get the usual cobordism group with oriented manifolds.

By the Thom-Pontrjagin theorem we have that in this case

$$
\mathcal{O}_{n}\left(\mathcal{F}, \mathcal{F}^{\prime}\right) \simeq \Omega_{k}\left(B N_{O(n-k)} \hat{\rho}(H) / \hat{\rho}(H)\right) \simeq \pi_{k}\left(M S O \wedge B N_{O(n-k)} \hat{\rho}(H) / \hat{\rho}(H)_{+}\right),
$$

the stable homotopy groups of the smash product of the Thom spectrum $M S O$ and $B N_{O(n-k)} \hat{\rho}(H) / \hat{\rho}(H)_{+}$.

We call two non-empty families $\mathcal{F}^{\prime} \subseteq \mathcal{F}$ adjacent if they differ by at most one representation in each dimension and quasi-adjacent if every element in $\mathcal{F}-\mathcal{F}^{\prime}$ is maximal in $\mathcal{F}$. In a more general way, since for different maximal representations the singular sets are closed disjoint sets, we can find disjoint tubular neighborhoods, and as before, identify the relative cobordism group as a sum of usual bordism groups of classifying spaces.

Theorem 2.11. Suppose that $\mathcal{F} \supset \mathcal{F}^{\prime}$ are quasi-adjacent. Then

$$
\mathcal{O}_{n}\left(\mathcal{F}, \mathcal{F}^{\prime}\right) \simeq \sum \Omega_{k_{j}, t}\left(B N_{O\left(n-k_{j}\right)} \hat{\rho}\left(H_{j}\right) / \hat{\rho}\left(H_{j}\right)\right)
$$

where $k_{j}$ is the multiplicity of the trivial representation in $\rho_{j}$ and the sum is over all isomorphism classes of elements $\left(H_{j}, \rho_{j}\right)$ of $\mathcal{F}(n)$ not in $\mathcal{F}^{\prime}(n)$.

A spectral sequence. If $\{e\}=\mathcal{F}_{0} \subseteq \mathcal{F}_{1} \subseteq \cdots \subseteq \mathcal{F}_{n}=\mathcal{F}$ is a sequence of families, the corresponding long exact sequences can be interpreted as an exact couple, giving a spectral sequence converging to $\mathcal{O}_{*}(\mathcal{F})$ with the $E^{1}$ term given by

$$
E_{p, q}^{1}=\mathcal{O}_{p+q}\left(\mathcal{F}_{p}, \mathcal{F}_{p-1}\right) .
$$

The filtration of $\mathcal{O}_{*}(\mathcal{F})$ is given,

$$
F_{p} \mathcal{O}_{n}(\mathcal{F}):=i m\left(\mathcal{O}_{n}\left(\mathcal{F}_{p}\right) \rightarrow \mathcal{O}_{n}(\mathcal{F})\right)
$$

and the differentials can be identified with the boundary map. The $E_{p, q}^{1}$ terms are computable (at least now it is a nonequivariant problem) if the families are quasi-adjacent.

The easiest way to construct these kind of filtrations is by adding at each step a representation of a new fixed group or use the canonical filtration given by the order $\leq$.

3. Orbifold cobordism in dimension 2 and 3. In this section we will use the machinery developed so far to calculate two and three dimensional cobordism groups of oriented and locally oriented orbifolds. By carefully choosing our families we will prove that the associated spectral sequences collapse at the $E^{2}$ term. In our spectral sequence the $E^{1}$-differentials are given by the boundary map and the following is the key geometric argument to show that it collapses. 
LEMMA 3.1. If an oriented orbifold $\mathcal{X}$ admits an orientation reversing involution, then it bounds.

Proof. Consider the action of $\mathbb{Z}_{2}$ on $\mathcal{X} \times I$ given by the product of the orientation reversing involutions on each term. $\left[\mathcal{X} \times I / \mathbb{Z}_{2}\right]$ is an oriented orbifold and the boundary is precisely $\mathcal{X}$.

To see that the spectral sequence collapses at the $E^{2}$-level we will use a local version of this idea.

Lemma 3.2. Suppose $(H, \rho)$ is a representation of maximal degree. Also assume that there is an $\alpha \in N_{O(n)} \rho(H)$ that is an orientation reversing involution. Consider the action of $H \rtimes \mathbb{Z}_{2}$ on $D(\rho) \times I$ given by

$$
(h, \alpha)(z, t)=(h \alpha z, \alpha t)
$$

where $\alpha$ acts on $I$ as multiplication by -1 .

Then the boundary of $\left[D(\rho) \times I / H \rtimes \mathbb{Z}_{2}\right]$ contains a copy of $[D(\rho) / H]$ and the complement has the local representations of the orbifold $\left[S(\rho) \times I / H \rtimes \mathbb{Z}_{2}\right]$.

An easy calculation shows that the local groups of $\left[S(\rho) \times I / H \rtimes \mathbb{Z}_{2}\right]$ are just the local groups of the action of the semidirect product $H \rtimes \mathbb{Z}_{2}$ on $S(\rho)$.

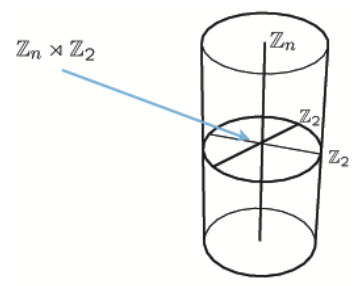

Fig. 1. Fixed sets of the action of $\mathbb{Z}_{n} \rtimes \mathbb{Z}_{2}$ on $D^{2} \times I$

In terms of our cobordism groups with restricted local representations, the previous lemma can be rephrased in the following way:

REMARK 3.3. In $\mathcal{O}\left(\left\{\left[D(\rho) \times I / H \rtimes \mathbb{Z}_{2}\right]\right\},\left\{\left[S(\rho) \times I / H \rtimes \mathbb{Z}_{2}\right]\right\}\right),[D(\rho) / H]$ and the boundary of $\left[D(\rho) \times I / H \rtimes \mathbb{Z}_{2}\right]$ represent the same element.

Two dimensional oriented orbifolds. Now we will construct a sequence of families of local representations to calculate the cobordism groups of two dimensional oriented orbifolds. For this, we need to know the possible local representations of oriented orbifolds. These are given by representations of cyclic groups. These representations have orientation reversing involutions given by complex conjugation, and the action of the semidirect product $\mathbb{Z}_{n} \rtimes \mathbb{Z}_{2}$ on $S^{1} \times I$ has two singular points with isotropy $\mathbb{Z}_{2}$.

This suggests one should take the families generated by the following representations. $\mathcal{F}_{0}=\{e\}$ the trivial family. The family $\mathcal{F}_{1}$ is generated by $\mathbb{Z}_{2}$ acting on $\mathbb{R}^{2}$ by the antipodal map. $\mathcal{F}_{2}$ the family generated by $\mathbb{Z}_{2} \rtimes \mathbb{Z}_{2}$ acting on $D^{2} \times I$ as in lemma 3.2.

Since, in general, the action of $\mathbb{Z}_{n} \rtimes \mathbb{Z}_{2}$ on $\partial\left(D^{2} \times I\right)$ has one singular point labelled $\mathbb{Z}_{n}$ with local representation given by the rotation action, then we have $\mathcal{F}_{1} \subseteq \mathcal{F}_{2}$. But 
also note that the local representations of the points of $\partial\left(D^{2} \times I\right)$ labeled $\mathbb{Z}_{2}$ are not orientation preserving.

The family $\mathcal{F}_{3}$ is generated by $\mathbb{Z}_{2} \rtimes \mathbb{Z}_{2}$ acting on $D^{2} \times I$ as in lemma 3.2 and the representations of $\mathbb{Z}_{n}$ acting on $\mathbb{R}^{2}$ by rotations. Finally, take $\mathcal{F}_{4}$ to be the family generated by the representations of $\mathbb{Z}_{n} \rtimes \mathbb{Z}_{2}$ acting on $D^{2} \times I$ as in lemma 3.2 , and as before $\mathcal{F}_{3} \subseteq \mathcal{F}_{4}$.

Then the adjacency conditions are satisfied, and we will see that the associated spectral sequence collapses at the $E^{2}$-level. Recall that the $E^{1}$-terms are given by $E_{p, q}^{1}=$ $\mathcal{O}_{p+q}\left(\mathcal{F}_{p}, \mathcal{F}_{p-1}\right)$ and that since the representations have degree zero, then every element of $\mathcal{O}_{p+q}\left(\mathcal{F}_{p}, \mathcal{F}_{p-1}\right)$ is a disk bundle over a finite set of points, i.e. a finite number of disks.

$E_{4,-2}^{1}=\mathcal{O}_{2}\left(\mathcal{F}_{4}, \mathcal{F}_{3}\right)$ is zero, because the two dimensional parts of the families $\mathcal{F}_{3}$ and $\mathcal{F}_{4}$ are the same. To see that $E_{3,-1}^{2}=0$, note that every element of $\mathcal{O}_{2}\left(\mathcal{F}_{3}, \mathcal{F}_{2}\right)$ is just a finite union of disks of representations of $\mathbb{Z}_{n}$, the differential

$$
d^{1}: E_{4,-1}^{1}=\mathcal{O}_{3}\left(\mathcal{F}_{4}, \mathcal{F}_{3}\right) \rightarrow E_{3,-1}^{1}=\mathcal{O}_{2}\left(\mathcal{F}_{3}, \mathcal{F}_{2}\right)
$$

is surjective by the choice of the family $\mathcal{F}_{4}$, this is precisely the content of remark 3.3.

The two dimensional parts of the families $\mathcal{F}_{2}$ and $\mathcal{F}_{1}$ have the same orientation preserving representations, and therefore $E_{2,0}^{1}=\mathcal{O}_{2}\left(\mathcal{F}_{2}, \mathcal{F}_{1}\right)$ is zero. To see that $E_{1,1}^{2}=0$, we need slightly more care, as before $E_{1,1}^{1}=\mathcal{O}_{2}\left(\mathcal{F}_{1}, \mathcal{F}_{0}\right)$ is generated by a disk representation of $\mathbb{Z}_{2}$. Now attach to the orbifold $\left[D^{2} \times I / \mathbb{Z}_{2} \rtimes \mathbb{Z}_{2}\right]$, which has three singular points labeled $\mathbb{Z}_{2}$, a handle cylinder $\left[D^{2} / \mathbb{Z}_{2}\right] \times I$ to get a three dimensional orbifold with boundary a manifold with only one singularity labeled $\mathbb{Z}_{2}$. This orbifold is an element of $\mathcal{O}_{3}\left(\mathcal{F}_{2}, \mathcal{F}_{1}\right)$ which is sent by the differential $d^{1}$ to the generator of $\mathcal{O}_{2}\left(\mathcal{F}_{1}, \mathcal{F}_{0}\right)$. Therefore $E_{1,1}^{2}=0$ as before. Since every two dimensional oriented manifold bounds, i.e. $F_{0} \mathcal{O}_{2}=0$, and we have seen that all $F_{p} \mathcal{O}_{2} / F_{p-1} \mathcal{O}_{2} \cong E_{p, 2-p}^{\infty}=0$, we have that,

THEOREM 3.4. Every two dimensional effective oriented orbifold bounds.

The proof also gives a procedure to construct the cobordism, take $\mathcal{X} \times I$ and to each point of singularity attach the orbifold $\left[D^{2} \times I / \mathbb{Z}_{n} \rtimes \mathbb{Z}_{2}\right]$, this gives a cobordism between $\mathcal{X}$ and an orbifold with an even number of points labeled $\mathbb{Z}_{2}$. Now these types of orbifolds are cobordant to manifolds by attaching orbifolds of the form $\left[D^{2} / \mathbb{Z}_{2}\right] \times I$, where $\mathbb{Z}_{2}$ acts only in the first factor as the antipodal map.

Three dimensional oriented orbifolds. Now we want to do the same in the three dimensional case. This is possible because we know the local structure of a three dimensional oriented effective orbifold. The finite subgroups of $S O(3)$ are given by cyclic, dihedral or the symmetries of a regular solid and the quotient orbifolds $\left[S^{2} / G\right]$ have underlying space $S^{2}$ with 2 or 3 cone points and are of the following form.

TheOrem 3.5 (Local structure in dimension three). A three dimensional locally oriented orbifold locally is a cone on one of the following local models.

- $S^{2}(n, n)$ if $G$ is cyclic of order $n$.

- $S^{2}(2,2, n)$ if $G=\mathbb{Z}_{n} \rtimes \mathbb{Z}_{2}$ is $D_{n}$, the dihedral of order $2 n$.

- $S^{2}(2,3,3)$ if $G=\mathcal{T}$, are the symmetries of regular tetrahedron.

- $S^{2}(2,3,4)$ if $G=\mathcal{O}$ are the symmetries of regular cube or octahedron. 
- $S^{2}(2,3,5)$ if $G=\mathcal{I}$ are the symmetries of regular icosahedron or dodecahedron.

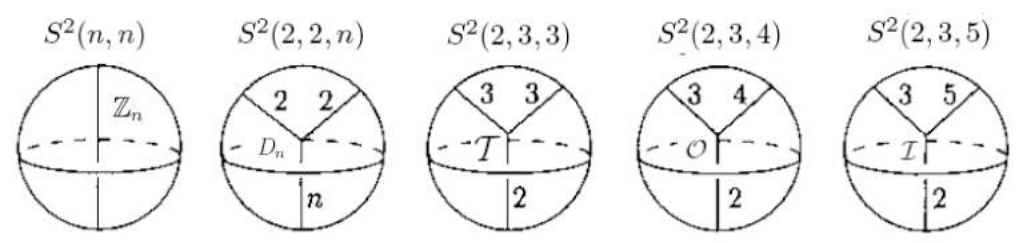

Fig. 2. Underlying spaces of the local models of three dimensional oriented orbifolds

See [4] or [10].

Note that since the dimension is odd, multiplication by -1 is an orientation reversing involution that commutes with any element of $S O(3)$, therefore we can consider

$$
\left[D^{3} \times I / G \times \mathbb{Z}_{2}\right]
$$

where the action is given by

$$
(h, \alpha)(v, t)=(h \alpha v, \alpha t) .
$$

The boundary of this orbifold has local representations the old ones and the ones as in lemma 3.2. These isotropy groups are given by the semidirect products

$$
G_{v} \rtimes \mathbb{Z}_{2}
$$

with $G_{v}$ the isotropy of an element of $\left[S^{2} / G\right]$. But note that these groups are just cyclic groups. Therefore the isotropy groups of these actions on $S^{2} \times I$ are given by dihedral groups.
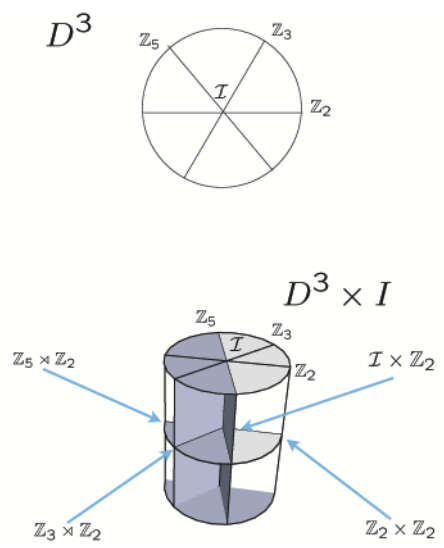

Fig. 3. Some fixed sets of the action of $\mathcal{I}$ on $D^{3}$ and of $\mathcal{I} \times \mathbb{Z}_{2}$ on $D^{3} \times I$

This suggests one should take the families generated by the following representations. $\mathcal{F}_{0}=\{e\}$ the trivial family. The family $\mathcal{F}_{1}$, generated by the representations of $\mathbb{Z}_{n}$ acting on $\mathbb{R}^{3}$ by rotations. The family $\mathcal{F}_{2}$, generated by $\mathbb{Z}_{n} \rtimes \mathbb{Z}_{2}$ acting on $D^{2} \times I$ as in lemma 3.2 .

The family $\mathcal{F}_{3}$ is generated by $\mathbb{Z}_{n} \rtimes \mathbb{Z}_{2}$ acting on $D^{2} \times I$ as in lemma 3.2 and the representations of the symmetries of the tetrahedron, octahedron and icosahedron acting 
on $\mathbb{R}^{3} . \mathcal{F}_{4}$ to be the family generated by the representations of the semidirect products $\mathcal{I} \times \mathbb{Z}_{2}, \mathcal{T} \times \mathbb{Z}_{2}, \mathcal{O} \times \mathbb{Z}_{2}$ on $D^{3} \times I$ as in lemma 3.2.

As in the two-dimensional case, this gives an increasing sequence of families of local representations and the adjacency conditions are satisfied. As before, we will see that the associated spectral sequence collapses at the $E^{2}$-level. $E_{4,-1}^{1}=\mathcal{O}_{3}\left(\mathcal{F}_{4}, \mathcal{F}_{3}\right)=0$ because $\mathcal{F}_{4}, \mathcal{F}_{3}$ agree on dimension 3. $\mathcal{F}_{4}$ was chosen to make the differential

$$
d^{1}: E_{4,0}^{1}=\mathcal{O}_{4}\left(\mathcal{F}_{4}, \mathcal{F}_{3}\right) \rightarrow E_{3,0}^{1}=\mathcal{O}_{3}\left(\mathcal{F}_{3}, \mathcal{F}_{2}\right)
$$

surjective, therefore $E_{3,0}^{2}=0$. The two-dimensional part of $\mathcal{F}_{1}$ consists of the twodimensional orientation preserving representations of $\mathbb{Z}_{n}$. The differential

$$
d^{1}: E_{2,1}^{1}=\mathcal{O}_{3}\left(\mathcal{F}_{2}, \mathcal{F}_{1}\right) \rightarrow E_{1,1}^{1}=\mathcal{O}_{2}\left(\mathcal{F}_{1}, \mathcal{F}_{0}\right)
$$

is injective, since any element of $\mathcal{O}_{3}\left(\mathcal{F}_{2}, \mathcal{F}_{1}\right)$ is a union of disks of dihedral representations, the boundaries of these disks are spheres that have one singularity $\mathbb{Z}_{n}$ and two $\mathbb{Z}_{2}$ 's, these spheres cannot bound in $\mathcal{O}_{2}\left(\mathcal{F}_{1}, \mathcal{F}_{0}\right)$. Thus $E_{2,1}^{2}=0$.

Now,

$$
E_{1,2}^{1}=\mathcal{O}_{3}\left(\mathcal{F}_{1}, \mathcal{F}_{0}\right)=\mathcal{O}_{3}\left(\mathbb{Z}_{n},\{e\}\right)=\Omega_{1, t}\left(B N_{O(2)} \mathbb{Z}_{n} / \mathbb{Z}_{n}\right)
$$

which is the cobordism group of orbivector bundles over 1-dimensional purely ineffective orbifolds, where we require the total space of the orbivector bundle to be oriented. Since any 1-dimensional compact closed manifold is naturally oriented, this group is the cobordism group of oriented orbivector bundles over one-dimensional purely ineffective orbifolds, i.e.

$$
\Omega_{1}\left(B\left(N_{S O(2)} \mathbb{Z}_{n} / \mathbb{Z}_{n}\right)\right) .
$$

But $S O(2)=S^{1}$ is commutative, thus $N_{S O(2)} \mathbb{Z}_{n}=S^{1}$ and

$$
B\left(N_{S O(2)} \mathbb{Z}_{n} / \mathbb{Z}_{n}\right)=B\left(S^{1} / \mathbb{Z}_{n}\right)=B S^{1}=\mathbb{C P}^{\infty} .
$$

Since $\mathbb{C P}^{\infty}$ is simply connected, $\Omega_{1}\left(B\left(N_{S O(2)} \mathbb{Z}_{n} / \mathbb{Z}_{n}\right)\right)=E_{1,2}^{1}$ is zero. Therefore all the $E^{2}$-terms are zero. By Rohlin's theorem, every three dimensional oriented manifold bounds, therefore,

Theorem 3.6 (K. Druschel). Every three dimensional effective oriented orbifold bounds.

The previous arguments can be used to study the cobordism groups of locally oriented orbifolds. Word by word we can make the corresponding definitions and the following is the corresponding version of theorem 2.11 .

TheOREM 3.7. Suppose that $\mathcal{F} \supset \mathcal{F}^{\prime}$ are families which differ at dimension $n$ by the representation $(H, \rho)$, i.e. $\mathcal{F}(n)-(H, \rho)=\mathcal{F}^{\prime}(n)$. Then the locally oriented cobordism group of effective orbifolds

$$
\mathcal{O}_{n}\left(\mathcal{F}, \mathcal{F}^{\prime}\right) \simeq \mathfrak{N}_{n}\left(B N_{O(n-k)} \hat{\rho}(H) / \hat{\rho}(H)\right)
$$

where $\mathfrak{N}_{n}\left(B N_{O(n-k)} \hat{\rho}(H) / \hat{\rho}(H)\right)$ is the usual (unoriented) bordism group.

As previously shown, the same spectral sequence argument shows that every locally oriented orbifold is cobordant to a locally oriented manifold. Now a locally oriented manifold is just an unoriented manifold. Thom completely calculated the cobordism ring 
of manifolds as the polynomial algebra $\mathbb{Z}_{2}\left[x_{i} \mid i \neq 2^{j}-1\right]$, in particular, every three dimensional manifold bounds, therefore

\section{THEOREM 3.8. Every three dimensional effective locally oriented orbifold bounds.}

Now, $\mathfrak{N}_{2} \cong \mathbb{Z}_{2}$ and the generator can be taken to be $\mathbb{R} \mathbb{P}^{2}$, a similar spectral sequence argument allows us to conclude that every two dimensional locally oriented orbifold is cobordant to a manifold. Although by an argument with Stiefel-Whitney numbers we know that $\mathbb{R P}^{2}$ it is not the boundary of another manifold, we still have to prove that is not the boundary of a locally oriented orbifold. For example it is the boundary of another orbifold, just consider the three dimensional disk $D^{3} \bmod$ the antipodal action. This is an orbifold with one singular point with isotropy $\mathbb{Z}_{2}$ and its boundary is the real projective plane. Indeed without any restriction, any orbifold is the boundary of another orbifold, just consider $\mathcal{X} \times\left[I / \mathbb{Z}_{2}\right]$, where $\mathbb{Z}_{2}$ acts by multiplying by -1 .

Now, even though we are only considering two-dimensional orbifolds we need to use families that will exhaust all possible three-dimensional orientation preserving ones to conclude that the real projective space does not bound. Take the families generated by the following representations: $\mathcal{F}_{0}=\{e\}$ the trivial family. The family $\mathcal{F}_{1}$ is generated by the representations of $\mathbb{Z}_{n}$ acting on $\mathbb{R}^{3}$ by rotations. $\mathcal{F}_{2}$ the family generated by $\mathbb{Z}_{n} \rtimes \mathbb{Z}_{2}$ acting on $D^{2} \times I$ as in lemma 3.2 and the family $\mathcal{F}_{3}$ generated by $\mathbb{Z}_{n} \rtimes \mathbb{Z}_{2}$ acting on $D^{2} \times I$ as in lemma 3.2 and the representations of the symmetries of the tetrahedron, octahedron and icosahedron acting on $\mathbb{R}^{3}$.

By induction, let us see that $\mathfrak{N}_{2} \rightarrow \mathcal{O}_{2}\left(\mathcal{F}_{i}\right)$ is injective. By theorem 3.7, the map $\mathfrak{N}_{2}=\mathcal{O}_{2}\left(\mathcal{F}_{0}\right) \rightarrow \mathcal{O}_{2}\left(\mathcal{F}_{1}\right)$ is injective if $\mathcal{O}_{3}\left(\mathcal{F}_{1}, \mathcal{F}_{0}\right) \cong \bigoplus \mathfrak{N}_{1}\left(B N_{O(2)} \mathbb{Z}_{n} / \mathbb{Z}_{n}\right) \rightarrow \mathfrak{N}_{2}$ is the zero map. But the elements of the relative group, which are disk orbibundles, are sent by the boundary map $\mathcal{O}_{3}\left(\mathcal{F}_{1}, \mathcal{F}_{0}\right) \rightarrow \mathfrak{N}_{2}$ to manifolds fibered over the circle, such manifolds have Euler characteristic zero and therefore cannot be cobordant as manifolds to $\mathbb{R P}^{2}$ which is the generator of $\mathfrak{N}_{2}$.

Since the adjacency conditions are satisfied, the group $\mathcal{O}_{3}\left(\mathcal{F}_{2}, \mathcal{F}_{1}\right)$ is generated by disks of representations, the boundary map sends these disks to one of the spheres described in theorem 3.5, and these spheres with an odd number of cyclic singularities cannot be cobordant to $\mathbb{R P}^{2}$ in $\mathcal{O}_{2}\left(\mathcal{F}_{1}\right)$. Therefore the map $\mathfrak{N}_{2} \rightarrow \mathcal{O}_{2}\left(\mathcal{F}_{2}\right)$ can be factored

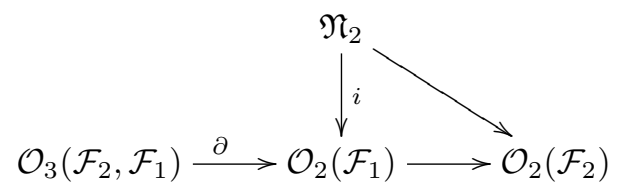

and is injective.

The three dimensional parts of $\mathcal{F}_{2}$ and $\mathcal{F}_{3}$ are the same and therefore $\mathcal{O}_{3}\left(\mathcal{F}_{3}, \mathcal{F}_{2}\right)=0$ and we conclude similarly that $\mathfrak{N}_{2} \rightarrow \mathcal{O}_{2}\left(\mathcal{F}_{3}\right)$ is injective.

THEOREM 3.9. $\mathbb{R P}^{2}$ generates the second cobordism group of effective locally oriented orbifolds.

As discussed above, without further restrictions on the local representations of the orbifolds that we allow, all the cobordism groups become zero. An intermediate case 
between all orbifolds and locally oriented orbifolds occurs when we restrict our groups to be of odd order. In this case a complete description of the cobordism ring as a module over $\mathfrak{N}$ can be given [1].

THEOREM 3.10. Denote by $\mathfrak{N}_{*, \text { orb }}^{\text {odd }}$ the cobordism ring of effective orbifolds with isotropy groups of odd order, then

$$
\mathfrak{N}_{*, \text { orb }}^{\text {odd }} \cong \mathfrak{N}_{*} \bigoplus_{H \text { odd }} \bigoplus_{\operatorname{Rep}_{*}(H)} \mathfrak{N}_{\operatorname{degree}(\rho)}\left(B N_{O(*-\operatorname{degree}(\rho))} H / H\right)
$$

where the sum extends over all groups of odd order and all faithful representations.

In particular, since all two dimensional faithful representations are given by cyclic groups and have degree zero, then

$$
\mathfrak{N}_{2, \text { orb }}^{\text {odd }} \cong \mathfrak{N}_{2} \bigoplus_{\mathrm{n} \text { odd }} \mathfrak{N}_{0}\left(B N_{O(2)} \mathbb{Z}_{n} / \mathbb{Z}_{n}\right) \cong \bigoplus_{\mathbf{n} \text { odd }} \mathbb{Z}_{2}
$$

where the generators can be taken to be the orbifold real projective spaces $\mathbb{R} \mathbb{P}^{2} / \mathbb{Z}_{n}$. In the three dimensional case, the only odd groups that can appear as isotropy groups of three dimensional orbifolds are the cyclic groups, thus

$$
\mathfrak{N}_{3, \text { orb }}^{\text {odd }} \cong \bigoplus_{\mathrm{n} \text { odd }} \mathfrak{N}_{1}\left(B N_{O(2)} \mathbb{Z}_{n} / \mathbb{Z}_{n}\right)
$$

An application of the Serre spectral sequence shows that each of these groups is isomorphic to $\mathfrak{N}_{1}(B O(2))$, which is $\mathbb{Z}_{2}$, therefore

$$
\mathfrak{N}_{3, \text { orb }}^{\text {odd }} \cong \bigoplus_{\mathbf{n} \text { odd }} \mathbb{Z}_{2}
$$

\section{References}

[1] A. Ángel, Orbifold cobordism, preprint, 2007.

[2] P. E. Conner and E. E. Floyd, Differentiable Periodic Maps, Ergebnisse der Mathematik und ihrer Grenzgebiete 33, Academic Press, New York, 1964.

[3] —, - Maps of odd period, Ann. of Math. (2) (1966), 143-156.

[4] D. Cooper, C. D. Hodgson and S. P. Kerckhoff, Three-dimensional orbifolds and conemanifolds, Mathematical Society of Japan, Tokyo, 2000.

[5] K. S. Druschel, Oriented orbifold cobordism, Pacific J. Math. 164 (1994), 299-319.

[6] - The cobordism of oriented three dimensional orbifolds, Pacific J. Math. 193 (2000), $45-55$.

[7] A. Henriques and D. S. Metzler, Presentations of noneffective orbifolds, Trans. Amer. Math. Soc. 356 (2004), 2481-2499.

[8] P. Iglesias, Y. Karshon and M. Zadka, Orbifolds as diffeologies, http://arXiv.org:math/ 0501093, 2005.

[9] C. Kosniowski, Actions of Finite Abelian Groups, Research Notes in Mathematics 18, Pitman (Advanced Publishing Program), Boston, Mass., 1978.

[10] G. Ramírez, I. Ana and J. Seade, Introduction to Classical Geometries, Birkhäuser, Basel, 2007. 